\title{
Spécificités de la mortalité et de son évolution dans les cantons entre 1920/21 et 1988/93
}

\section{Résumé}

Au cours du siècle, le recul de la mortalité ne s'est pas fait au même rythme dans l'ensemble des cantons. Cette évolution différente a mené à une forte réduction des disparités de la mortalité. Ainsi, alors qu'en 1920/21 une typologie cantonale permettait de regrouper statistiquement les cantons selon les risques de décès aux âges infantiles, juvéniles ainsi qu'aux âges mûrs, cette classification n'est plus possible aujourd'hui. Le présent article décrit cette évolution différentielle et montre que, d'une situation dans laquelle les villes et les régions industrialisées étaient favorisées en terme d'espérance de vie à la naissance - principalement en raison de la faible mortalité infantile -, nous sommes passé à un renversement de la situation, dicté par les disparités de la mortalité aux âges adultes et avancés.

\section{Introduction}

L'allongement de la durée de vie a été considérable au cours du siècle. En cent ans, l'espérance de vie à la naissance a passé de 45,7 ans (hommes) et 48,5 ans (femmes) à 74,2 ans, resp. 81,0 ans. Les conséquences sur la composition de la population selon l'âge et le sexe sont importantes (HÖPFINGER 1995) et le seront encore ces prochaines décennies. Pour donner un exemple, en 1900 , la proportion de la population âgée de 65 ans et plus, par rapport à la population totale, était proche de $5,8 \%$, contre $14,8 \%$ en 1995 . Cette valeur devrait atteindre $24,9 \%$ en 2050 selon les scénarios de l'OFS (OFS 1996a).

En contribuant au vieillissement de la population, l'allongement de la durée de vie n'a pas eu seulement une influence sur la structure par âge de la population. Il a passablement modifié le comportement individuel, l'organisation des années passées à la retraite, le fonctionnement et les caractéristiques de la société : Ainsi, il a influencé le comportement des personnes dans des domaines aussi différents que la consommation, le recours aux soins, les investissements ou les transferts intergénérationnels. Il a aussi eu des effets profonds sur l'organisation de la prévoyance sociale et sur l'économie, ainsi que sur les opinions et les normes socio-culturelles (Commission fédérale Vieillir en Suisse 1995; HAUG 1995).

Les disparités régionales ou internationales en matière de durée moyenne de vie apportent des informations importantes pour la connaissance du phénomène de la mortalité ainsi que pour la gestion de la santé publique. Notamment, pour l'évaluation des buts de la stratégie européenne de la Santé pour tous, l'OMS a proposé l'utilisation d'indicateurs de la mortalité pour 7 des 12 buts à atteindre afin d'améliorer l'état de santé de la population (OMS 1985). En Suisse, plusieurs recherches ont par ailleurs montré, en comparant les risques de décès entre les cantons, les spécificités régionales de la mortalité pour différentes causes et mis en évidence certains problèmes prioritaires de santé publique (voir notamment OFS 1987). Pourtant, alors que les analyses comparatives de la mortalité au niveau cantonal se sont multipliées au cours des dernières années (WANNER et al. à paraître; OFS 1997a; OFS 1996b; OFS 1987; OFS 1984), une analyse de la dynamique des progrès et de l'évolution des disparités pour l'ensemble du siècle n'a jamais été effectuée en Suisse, contrairement à plusieurs autres pays (CASELLI et al. 1995; ANSON 1993; KUNST et al. 1991). Une telle étude portant sur l'ensemble du siècle améliorerait notre compréhension du phénomène de la mortalité et de la transition d'un niveau de mortalité élevée à un niveau de mortalité faible. Elle autoriserait par ailleurs l'élaboration d'hypothèses sur les tendances futures de la mortalité.

Le présent article a pour but de décrire les évolutions cantonales en matière de mortalité depuis la fin de la Première Guerre mondiale. L'utilisation d'outils de statistiques multivariées décrit les principales caractéristiques de la mortalité en Suisse et dans les cantons au cours du XXe siècle. Des méthodes démographiques interprètent l'évolution de la mortalité, notamment en recourant à une décomposition des différences observées entre plusieurs cantons. Cette décomposition attribue les différences entre deux tables de mortalité selon les classes d'âges qui y contribuent. L'application de ces deux familles de méthodes permet une discussion sur l'évolution de la mortalité et des disparités cantonales.

\section{Période étudiée}

Les statistiques disponibles à une échelle cantonale permettent d'élaborer des tables de mortalité pour les années centrées autour des recensements. Ceux-ci ont

Peng Fei, Philippe Wanner, Stéphane Cotter, Office fédéral de la statistique, section de l'évolution de la population, Schwarztorstraße 53, 3003 Berne. 
lieu en Suisse tous les dix ans. Nous nous sommes limités à trois dates $(1920 / 21,1969 / 72$ et 1988/93'1), définissant deux périodes distinctes d'évolution. Le choix de ces dates se justifie par le contexte sanitaire observé en Suisse et, plus généralement, en Europe.

Ainsi, la période située entre 1920/21 et 1969/72 se caractérise par un important progrès socio-économique, sanitaire et en matière de conditions d'hygiène. Elle correspond à une forte diminution (ou transition) de la mortalité (voir notamment: CASELLI et al. 1995; CASELLI 1991; CASELLI et EGIDI 1991; OMRAN 1982). Les progrès sanitaires et sociaux durant cette période ont eu les conséquences suivantes:

a) une très forte diminution de la mortalité pour les maladies infectieuses et spécialement la tuberculose (GUBÉRAN 1979 et 1980), dont la diminution coöncide avec les premières utilisations de la streptomycine et du rimifon,

b) une baisse de la mortalité infantile due à la maîtrise des infections digestives ou respiratoires et des maladies infantiles classiques (rougeole, diphtérie), notamment en raison de l'amélioration des conditions d'hygiène et de l'alimentation des nourrissons,

c) la quasi-disparition de la mortalité maternelle.

L'essor économique qui a suivi la fin de la Seconde Guerre mondiale et ses conséquences sur les conditions de vie ont par ailleurs favorisé la survie des personnes d'âge avancé. Cette période a aussi coïncidé avec l'apparition à tous les âges d'une surmortalité des hommes par rapport aux femmes.

La période comprise entre 1969/72 et 1988/93 a été caractérisée par l'émergence d'un nouveau profil de mortalité selon la cause de décès, avec notamment une augmentation de la proportion des décès provoqués par les comportements à risque adoptés par la population depuis la fin de la guerre (en matière d'alimentation et de consommation d'alcool et de tabac principalement) et par l'apparition du SIDA. Ces évolutions défavorables contrastent avec l'amélioration des connaissances thérapeutiques et le développement des technologies médicales, qui ont eu pour conséquence de diminuer encore le risque de décès infantile et juvénile par une médecine de type curative et d'allonger la survie des personnes âgées par une médecine de type gériatrique. Ainsi, la période récente s'est caractérisée par une très forte diminution de la mortalité avant 20 ans et après 40 ans qui contraste avec une augmentation de la mortalité des jeunes adultes.

\section{Méthodes utilisées}

\subsection{Calcul des tables de mortalité et estimation de l'espérance de vie à la naissance}

Nous avons utilisé les tables de mortalité par sexe et par canton, calculées dans le cadre des travaux de l'Office fédéral de la statistique (OFs 1996b). Pour des raisons associées à la taille des populations et au faible nombre de décès observés, les cantons de Suisse centrale (Uri,
Schwyz, Obwald, Nidwald et Glaris) ont été regroupés, de même que les deux demi-cantons d'Appenzell et ceux de Zoug et de Schaffhouse. Pour le canton du Jura, seule la dernière table est disponible. Auparavant, le territoire du dernier-né des cantons suisses était compris dans celui de Berne.

L'espérance de vie à la naissance est utilisée comme indicateur de la mortalité générale. Elle représente le nombre moyen d'années à vivre pour une personne soumise au cours de sa vie aux conditions de la mortalité du moment. Le calcul de cet indicateur nécessite quelques hypothèses sur l'âge exact auquel survient le décès. La répartition des durées de vie des enfants décédés durant leur première année de vie a été effectuée par une méthode dite de SHYROCK et SIEGEL (1971), qui répartit les décès selon la durée de vie écoulée depuis la naissance en fonction du niveau de mortalité infantile. La répartition des décès entre 1 et 4 ans a été estimée en recourant aux tables-types de mortalité, famille Ouest, construits par COALE et DEMENY (1966). A partir du groupe d'âges 5-9 ans, nous avons supposé une répartition linéaire des décès au sein de chaque groupe d'âges.

Les quotients (ou probabilités) de mortalité pour différents groupes d'âges ont également été considérés dans notre analyse: il s'agit des âges 0 an, 1-4 ans, 5-9 ans, 10-14 ans et ainsi de suite jusqu'à l'extinction de la table (95 ans et plus).

Nous obtenons ainsi, pour chaque date et sexe, une matrice de 19 lignes ou observations (cantons ou regroupements de cantons) et 22 colonnes ou indicateurs (quotients de mortalité et espérance de vie à la naissance) pour les deux premières séries de tables, et de 20 lignes (avec le Jura) et 22 colonnes pour la dernière série de tables.

\subsection{Méthodes statistiques multivariées: analyse en composantes principales et analyse de classification}

L'interprétation d'informations regroupées sous forme d'une matrice comprenant plus de 400 valeurs n'est pas aisée. Afin de réduire le nombre de variables et regrouper les informations tout en conservant une part élevée de la variance, nous avons effectué une analyse statistique multivariée, s'appuyant d'une part sur une analyse de classification hiérarchique et d'autre part sur une analyse en composantes principales. L'analyse de classification a été menée pour chaque période et pour chaque sexe à l'aide de la procédure CLUSTER du logiciel SAS (SAS Institute Inc. 1989). Elle définit des ressemblances cantonales et regroupe les cantons en fonction de leurs caractéristiques de mortalité. Selon les cas, entre 4 et 5 groupes de cantons ont été retenus. L'analyse en composantes principales (ACP - VOLLÉ 1985) a été effectuée pour résumer en un nombre limité de facteurs les variables de la mortalité. Dans notre cas, 2 facteurs permettent de traduire les principaux schémas de la mortalité en conservant une part élevée de la variance. Cette variance a été répartie par une rotation par la pro- 
cédure FACTOR, méthode VARIMAX (SAS Institute Inc. 1989).

Les deux méthodes peuvent être comparées par une confrontation graphique des cantons et regroupements de cantons sur le premier plan factoriel défini par les deux premières composantes de l'ACP.

Cette analyse statistique a été menée pour 6 séries de tables ( 3 dates et 2 sexes). L'ACP a également été effectuée pour les trois périodes considérées ensemble, afin de mesurer le déplacement temporel des cantons sur les plans factoriels. L'évolution des schémas de mortalité durant le temps est alors résumé.

\subsection{Méthodes démographiques: rythme de diminution de la mortalité et décomposition des différences cantonales}

Les méthodes utilisées ont été discutées en détail dans le contexte de la Suisse au cours d'une analyse des différences de mortalité entre hommes et femmes (PENG FEI et al. 1997) et sur l'évolution de la mortalité cantonale au cours des 10 dernières années (WANNER et al. 1997). En premier lieu, un indicateur du rythme de déclin de la mortalité, indépendant de la valeur initiale, exprime le changement relatif de la durée de vie vécue. Le rythme du déclin de la mortalité est mesuré par la relation suivante (ARRIAGA, 1984):

$$
V_{t, t+n}=\frac{e_{0}^{t+n}-e_{0}^{t}}{100-e_{0}^{t}} \times 100
$$

où $e f$ représente l'espérance de vie à la naissance au temps $t$.

Dans une seconde étape, nous avons distribué les différences d'espérance de vie observées, entre deux cantons, selon la contribution de différentes classes d'âges. Plusieurs méthodes de distribution existent (VALKOVICS 1996). Nous avons employé la méthode d'ARRIAGA (1984).

ARRIAGA a développé une technique de décomposition des différences d'espérance de vie entre deux tables, qui permet de répartir ces différences selon le type d'impact sur l'espérance de vie. L'impact total de la différence de la mortalité entre deux cantons dans un groupe d'âges sur la différence de l'espérance de vie $\left({ }_{i} T E_{x}\right)$ peut être divisé en trois parts: l'impact direct $\left({ }_{i} D E_{x}\right)$, l'impact indirect $\left({ }_{i} I E_{x}\right)$ et l'impact interactif $\left({ }_{i} I_{x}\right)$. Afin de bien saisir ce que l'auteur entend avec ces trois notions, il faut avoir en tête que l'espérance de vie (ou la différence entre l'espérance de vie de deux tables) est la somme des années de vie vécues après l'âge $x$ étudié. Ainsi, si on considère deux tables strictement identiques à l'exception de la probabilité de survie entre deux âges, par exemple entre 15 et 19 ans révolu, on observera que le nombre d'années de vie vécues entre 15 et 19 ans sera différent pour les deux populations (ce que l'auteur appelle l'impact direct). En plus, comme le nombre de survivants à l'âge de 20 ans exact sera également différent, le nombre d'années de vie vécues après 20 ans variera également, même si le niveau de mortalité après 20 ans reste constant pour les deux tables (ce
qu'ARRIAGA appelle l'impact indirect). Si un second groupe d'âges connaît une variation de la mortalité (par exemple le groupe 20-24 ans), cette nouvelle variation aura elle-même un effet sur l'impact indirect provoqué par le groupe d'âges 15-19 ans. C'est l'impact interactif.

Pour chaque groupe d'âges, la somme des trois impacts est égal à l'impact total, valeur qui est présentée dans le présent article. Cette relation peut donc être exprimée ainsi:

$$
{ }_{i} T E_{x}={ }_{i} D E_{x}+{ }_{i} I E_{x}+{ }_{i} I_{x}
$$

L'impact direct est défini comme l'influence de la différence de mortalité au sein d'un groupe d'âges sur la différence de la durée de vie moyenne au sein de ce même groupe d'âges $(x, x+i)$, pour deux populations popl et $p o p 2$. Il peut être calculé par la formule suivante:

$$
{ }_{i} D E_{x}=\frac{l_{x}^{\text {pop1 }}}{l_{0}^{\text {popl }}} \times\left[{ }_{i} e_{x}^{\text {pop2 }}-{ }_{i} e_{x}^{\text {pop } 1}\right]
$$

où ${ }_{i} e_{x}$ représente l'espérance de vie entre l'âge $x$ et $x+i$, $l_{x}$ représente le nombre de survivants à l'âge $x$ et $l_{0}$ représente le nombre de survivants à l'âge 0 an (effectif ou racine de la table de mortalité).

L'impact indirect représente l'influence de la différence de mortalité au sein d'un groupe d'âges sur la disparité de la durée de vie aux groupes d'âges qui suivent tet peut être calculé par la relation:

$$
\begin{gathered}
\text {,IE }=\frac{{ }_{1} C S_{x}}{l_{a}^{\prime}} \times e_{x+i}^{\text {popl } 1} \\
\text { où: } \quad{ }_{i} C S_{x}=\left(l_{x}^{\text {popl }} \times \frac{l_{x+1}^{\text {pop } 2}}{l_{x}^{\text {pop } 2}}\right)-l_{x+1}^{p o p 1}
\end{gathered}
$$

L'impact interactif est calculé à partir de la formule:

$$
{ }_{i} I_{x}=\frac{{ }_{1} C S_{x}}{l_{a}^{p o p 1}} \times\left(e_{x+i}^{\text {pop } 2}-e_{x+i}^{\text {popl }}\right)
$$

Pour le dernier groupe d'âges ouvert, il n'existe plus d'impact indirect ni d'impact interactif, l'impact direct est calculé à l'aide de la relation:

$$
D E_{x+}=\frac{l_{x}^{p o p 1}}{l_{a}^{p o p 1}} \times\left(e_{x}^{\text {pop2 }}-e_{x}^{\text {pop } 1}\right)
$$

\subsection{Complémentarité des méthodes employées}

La présentation des niveaux d'espérance de vie à la naissance et l'étude des rythmes d'évolution apportent une vision descriptive des progrès enregistrés au cours des deux périodes étudiées. L'approche statistique est une démarche de regroupement ou de réduction de l'information disponible, d'une part par une classification des cantons et d'autre part à l'aide d'une transformation des quotients de mortalité par classe d'âge en plusieurs 
facteurs résumant un maximum d'informations. Cette approche part donc d'un nombre élevé de données pour ne retenir que des caractéristiques essentielles. L'approche démographique suit un cheminement inverse. A partir d'un indicateur résumé, l'espérance de vie à la naissance, on calcule des écarts de niveau ou d'évolution selon les cantons, écarts qui sont ensuite répartis selon les classes d'âges qui y contribuent. Par sa nature, cette décomposition permet une approche exploratoire des caractéristiques régionales. Les méthodes employées retenues sont donc complémentaires et permettent une description pertinente des schémas cantonaux de mortalité.

\section{Résultats}

\subsection{Comparaisons intercantonales}

Les valeurs cantonales de l'espérance de vie à la naissance sont présentées pour les trois dates étudiées (tableau 1; figures 1 et 2). En 1920/21 et 1968/73, les cantons à dominance urbaine bénéficiaient d'un schéma de mortalité plutôt favorable. Pour les femmes en 1920/21, le canton de Bâle-Ville se caractérisait par la durée de vie à la naissance la plus élevée (62,3 ans). A l'autre extrémité, le Tessin avait la durée de vie la plus faible
(50,4 ans). Pour les hommes, toujours en 1920/21, on retrouve un schéma similaire à celui des femmes. Neuchâtel avait l'espérance de vie la plus élevée $(58,4$ ans) et le Tessin la plus faible (48,2 ans).

En 1968/73, les cantons de Genève et du Valais se caractérisaient respectivement par la durée de vie moyenne la plus et la moins importante, tant pour les femmes (77,0 ans et 74,4 ans) que pour les hommes ( 71,1 ans et 68,2 ans). Les différences entre les valeurs extrêmes ont fortement diminué, puisqu'elles ne dépassaient alors pas 3 années de vie contre plus de 10 ans en 1920/21.

En 1988/93, les disparités de mortalité ont encore été réduites. Les cantons urbains ne bénéficiaient plus d'un niveau de mortalité favorable par rapport aux autres cantons, mais plutôt une surmortalité. Le Tessin rencontrait la durée de vie moyenne la plus élevée pour les femmes ( 81,8 ans) alors que Bâle-Ville rencontre la durée de vie la plus faible (79,8 ans). Ainsi, en 70 ans, les positions occupées par ces deux cantons se sont inversées. Pour les hommes on retrouve pratiquement le même schéma spatial que pour les femmes. Zoug et Schaffhouse d'une part (74,9 ans) et de Fribourg d'autre part (72,7 ans) se situent aux valeurs extrêmes.

Tant chez les femmes que chez les hommes et pour les deux périodes étudiées, le canton du Tessin s'est caractérisé à la fois par le rythme de déclin de la mortalité

Tableau 1: Espérance de vie à la naissance en Suisse et dans les cantons entre 1920/21 et 1988/93

\begin{tabular}{lcccccc}
\hline & \multicolumn{2}{c}{$1920 / 21$} & \multicolumn{2}{c}{$1969 / 72$} & \multicolumn{2}{c}{$1988 / 93$} \\
Canton & Hommes & Femmes & Hommes & Femmes & Hommes & Femmes \\
\hline Ensemble de la Suisse & 54,5 & 57,5 & 70,3 & 76,2 & 74,2 & 81,0 \\
Zurich & 56,6 & 60,2 & 70,9 & 76,9 & 74,2 & 80,8 \\
Berne & 56,7 & 59,6 & 70,4 & 76,4 & 74,4 & 81,2 \\
Lucerne & 52,8 & 56,5 & 70,0 & 76,0 & 74,6 & 81,2 \\
Uri, Schwyz, Obwald, Nidwald, Glaris & 52,2 & 54,7 & 69,4 & 75,3 & 74,3 & 80,8 \\
Zoug, Schaffhouse & 56,2 & 59,8 & 70,8 & 76,8 & 74,9 & 81,2 \\
Fribourg & 48,9 & 52,2 & 68,3 & 74,6 & 72,7 & 80,6 \\
Soleure & 54,6 & 57,5 & 70,0 & 75,9 & 74,5 & 80,7 \\
Bâle-Ville & 57,9 & 62,3 & 71,0 & 76,9 & 73,1 & 79,8 \\
Bâle-Campagne & 57,2 & 60,6 & 70,3 & 76,3 & 74,8 & 80,9 \\
Appenzell & 54,3 & 57,2 & 70,0 & 75,9 & 74,2 & 80,8 \\
Saint-Gall & 54,4 & 57,4 & 70,0 & 75,9 & 74,1 & 81,2 \\
Grisons & 54,0 & 56,0 & 69,6 & 75,3 & 73,9 & 80,9 \\
Argovie & 55,3 & 58,2 & 70,2 & 76,2 & 74,3 & 80,9 \\
Thurgovie & 54,3 & 57,6 & 69,5 & 75,4 & 74,5 & 81,3 \\
Tessin & 48,2 & 50,4 & 68,7 & 74,9 & 74,8 & 81,8 \\
Vaud & 56,0 & 59,1 & 70,5 & 76,7 & 74,3 & 81,3 \\
Valais & 48,9 & 51,5 & 68,2 & 74,4 & 73,0 & 81,0 \\
Neuchâtel & 58,4 & 60,6 & 70,0 & 75,8 & 73,8 & 81,0 \\
Genève & 56,1 & 58,6 & 71,1 & 77,0 & 74,6 & 81,7 \\
Jural & - & - & - & - & 73,2 & 80,4 \\
\hline
\end{tabular}

' Jusqu'en 1969/72, compris dans le canton de Berne 
K1 Lebenserwartung bei Geburt, 1988/93

Espérance de vie à la naissance, $1988 / 93$

In Jahren / En années



Fig. 1 L'état de la mortalité dans les cantons suisses en 1988/93. Espérance de vie à la naissance, hommes.

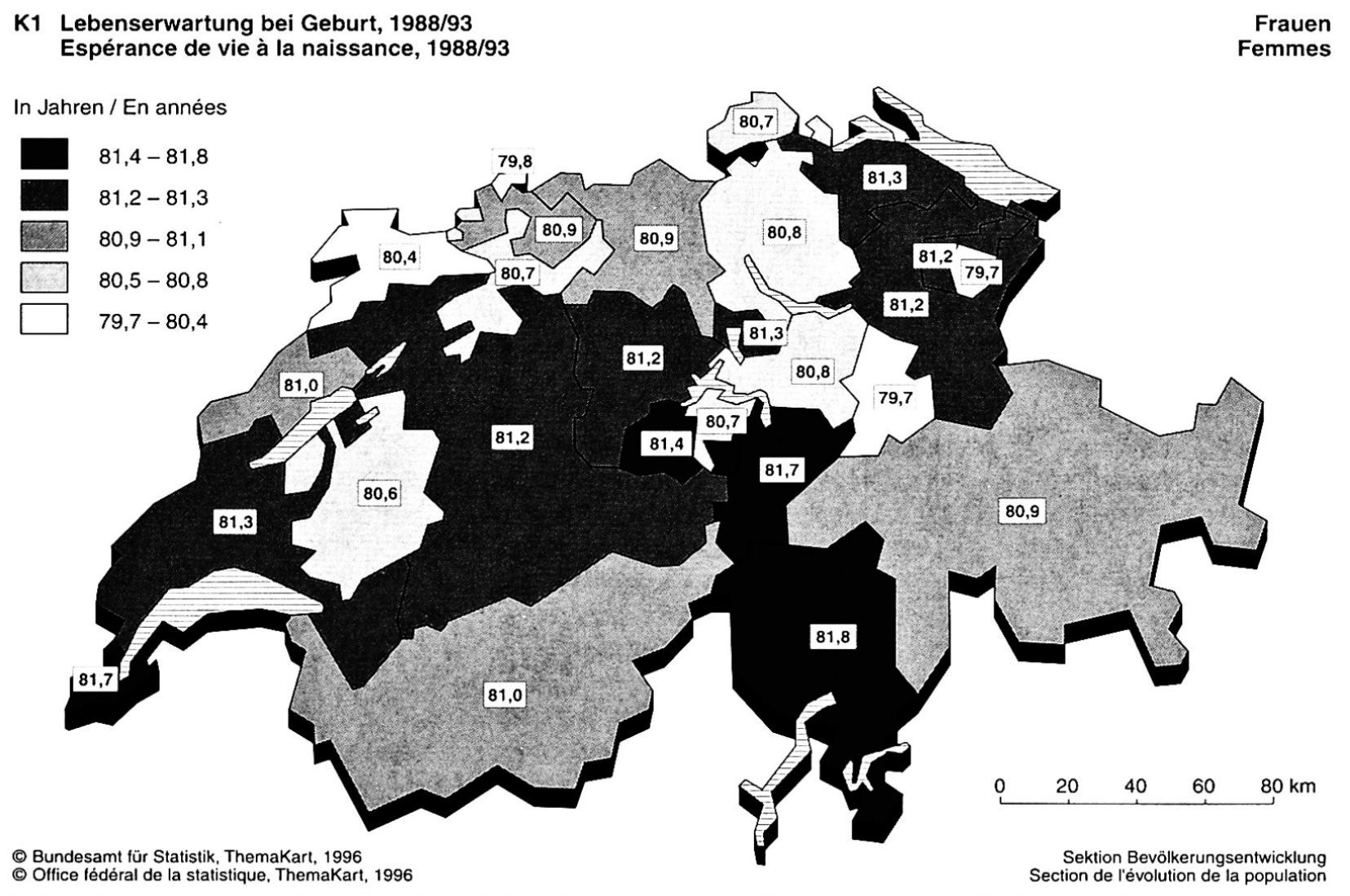

Fig. 2 L'état de la mortalité dans les cantons suisses en 1988/93. Espérance de vie à la naissance, femmes. 
le plus rapide et le gain le plus important de durée de vie (tableau 2). A l'opposé, entre 1920/21 et 1969/72, le canton de Neuchâtel a connu le déclin le moins rapide. Durant la deuxième période étudiée, Neuchâtel (hommes) et Bâle-Ville (femmes) ont connu le plus faible déclin de la mortalité. Entre 1969/72 et 1988/93, les Tessinois de sexe masculin ont augmenté de 6,1 ans leur espérance de vie à la naissance, contre seulement 3,5 ans pour les Genevois et 3,8 ans pour les résidants de Neuchâtel.

\subsection{Analyse statistique}

La confrontation des deux analyses multidimensionnelles (ACP et analyse de classification) est présentée dans les figures 3 à 8 . Cette confrontation définit les caractéristiques de la mortalité pour chaque groupe de cantons et présente visuellement les différentes structures de la mortalité observables en Suisse à 3 moments donnés. En plus, nous représentons une synthèse géométrique des disparités spatiales de la mortalité suisse durant le XXe siècle en prenant en considération les trois dates (figures 9 et 10).

Le regroupement des cantons par l'analyse de classification permet de conserver un pourcentage de variance relativement élevé pour les deux premières dates (entre $84 \%$ et $92 \%$ selon le sexe). En 1920/21, le résultat du regroupement est identique quel que soit le sexe (figures 3 et 4 ), signifiant ainsi que la structure cantonale obtenue s'applique tant chez les hommes que chez les femmes. Deux facteurs (l'un traduisant la mortalité aux âges élevés, l'autre la mortalité infantile) suffisent pour prendre en compte plus de $98 \%$ de la variance totale. Neuchâtel se distingue des autres cantons par une très faible mortalité tant infantile qu'aux âges avancés. A l'opposé, le Valais et le Tessin se caractérisent par une forte mortalité infanto-juvénile, et les cantons du centre de la Suisse, de Lucerne et de Fribourg par une forte mortalité aux âges avancés. Ces deux groupes de cantons ne sont pas très homogènes si on en croit la valeur du RMSSTD ${ }^{2}$. Les autres cantons se répartissent en deux groupes situés à une distance intermédiaire des trois autres classes de canton.

En 1969/72, le regroupement varie selon le sexe, néanmoins quelques caractéristiques régionales demeurent (Figures 5 et 6). Les résultats de l'ACP sont satisfaisants, deux facteurs permettent de prendre en compte plus de $98 \%$ de la variance totale. Les facteurs ont la même signification qu'en 1920/21 et traduisent respectivement la mortalité infanto-juvénile et la mortalité aux âges avancés. Avec une faible mortalité des jeunes et une forte mortalité des personnes âgées, Bâle-Campagne se différencie des autres cantons. Les cantons du Valais et de Fribourg se caractérisent par une forte mortalité des

Tableau 2: Augmentation de l'espérance de vie (en années) et progrès (rythme en \%) en Suisse et dans les cantons, de 1920/21 à 1988/93

$$
\text { entre } 1920 / 21 \text { et } 1969 / 72
$$
entre $1969 / 72$ et $1988 / 93$

Hommes

Femmes

Hommes

Femmes

\begin{tabular}{lcccccccc} 
Canton & années & $\%$ & années & $\%$ & années & $\%$ & années & $\%$ \\
\hline Ensemble de la Suisse & 15,8 & 44,1 & 18,7 & 56,5 & 3,9 & 18,7 & 4,8 & 29,7 \\
Zurich & 14,3 & 42,3 & 16,8 & 54,8 & 3,4 & 16,6 & 4,0 & 25,1 \\
Berne & 13,7 & 40,6 & 16,9 & 54,1 & 4,1 & 19,7 & 4,9 & 29,7 \\
Lucerne & 17,2 & 45,8 & 19,5 & 57,0 & 4,5 & 21,4 & 5,3 & 32,2 \\
Uri, Schwyz, Obwald, & & & & & & & & \\
Nidwald, Glaris & 17,1 & 45,0 & 20,6 & 57,3 & 4,9 & 22,8 & 5,6 & 33,0 \\
Zoug, Schaffhouse & 14,6 & 42,7 & 17,1 & 55,1 & 4,1 & 19,9 & 4,4 & 27,9 \\
Fribourg & 19,4 & 46,8 & 22,3 & 58,3 & 4,5 & 19,8 & 6,2 & 35,2 \\
Soleure & 15,4 & 43,1 & 18,5 & 55,6 & 4,5 & 21,1 & 4,7 & 28,1 \\
Bâle-Ville & 13,2 & 40,4 & 14,6 & 51,1 & 2,1 & 10,1 & 3,0 & 18,1 \\
Bâle-Campagne & 13,1 & 39,6 & 15,7 & 52,1 & 2,8 & 13,8 & 4,5 & 27,6 \\
Appenzell & 15,8 & 43,7 & 18,7 & 55,8 & 4,7 & 22,3 & 5,0 & 29,4 \\
Saint-Gall & 15,6 & 43,3 & 18,5 & 55,5 & 4,3 & 20,1 & 5,3 & 31,8 \\
Grisons & 15,6 & 42,9 & 19,3 & 55,6 & 4,4 & 20,4 & 5,7 & 33,6 \\
Argovie & 14,9 & 42,4 & 18,0 & 55,3 & 4,2 & 20,0 & 4,7 & 29,1 \\
Thurgovie & 15,1 & 42,0 & 17,8 & 53,8 & 2,8 & 13,3 & 5,7 & 33,5 \\
Tessin & 20,5 & 48,7 & 24,5 & 61,0 & 6,1 & 26,9 & 6,8 & 38,0 \\
Vaud & 14,5 & 42,2 & 17,6 & 55,5 & 3,8 & 18,0 & 4,8 & 29,1 \\
Valais & 19,4 & 46,8 & 22,9 & 58,5 & 4,9 & 21,2 & 6,7 & 37,4 \\
Neuchâtel & 11,6 & 36,2 & 15,2 & 50,5 & 3,8 & 17,8 & 5,1 & 30,1 \\
Genève & 15,0 & 43,6 & 18,4 & 57,2 & 3,5 & 17,0 & 4,7 & 28,5 \\
\hline
\end{tabular}




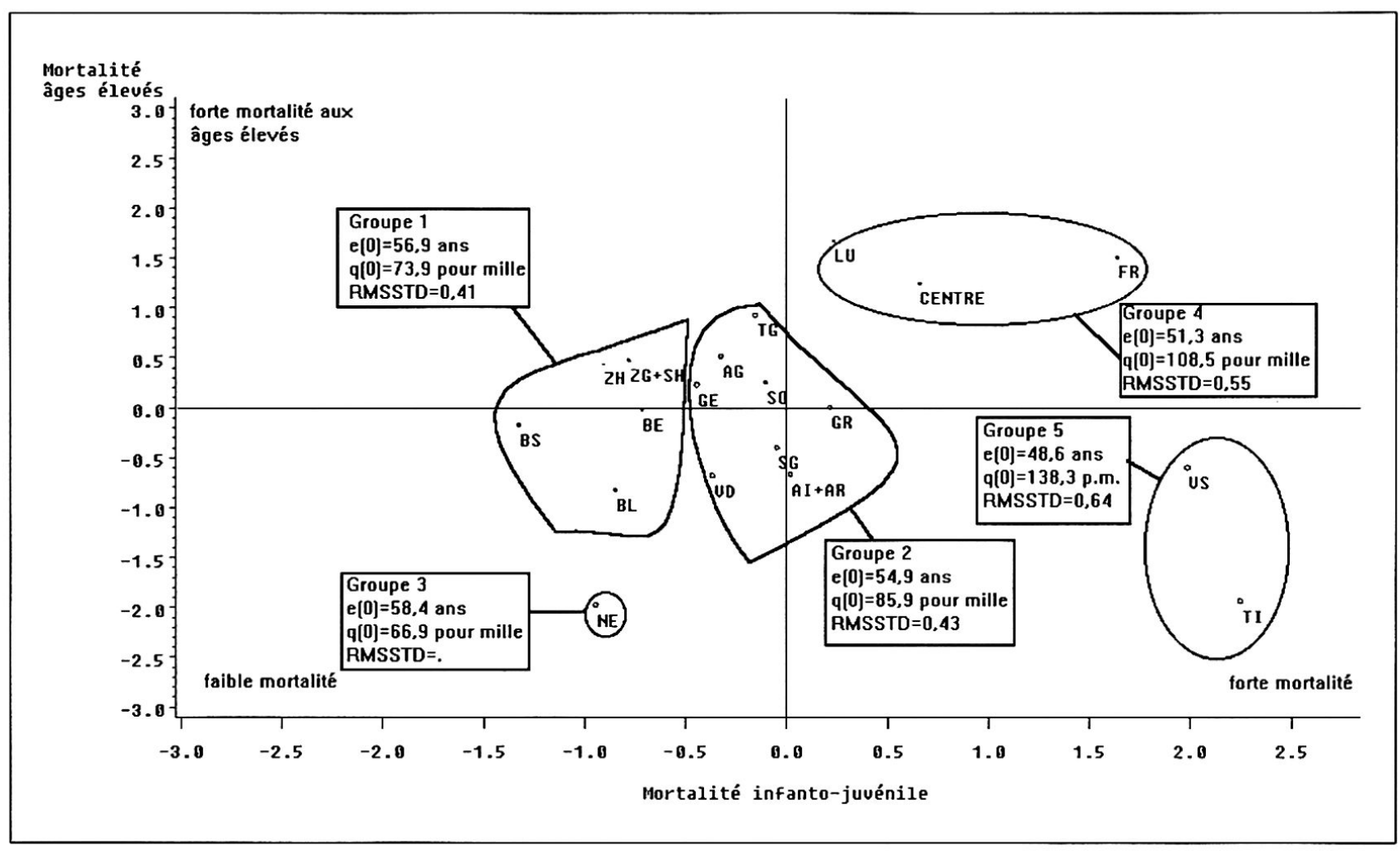

Fig. 3 Confrontation des résultats de l'analyse en composantes principales et de la classification. Position des cantons et groupes de cantons sur le premier plan factoriel, hommes, 1920/21.

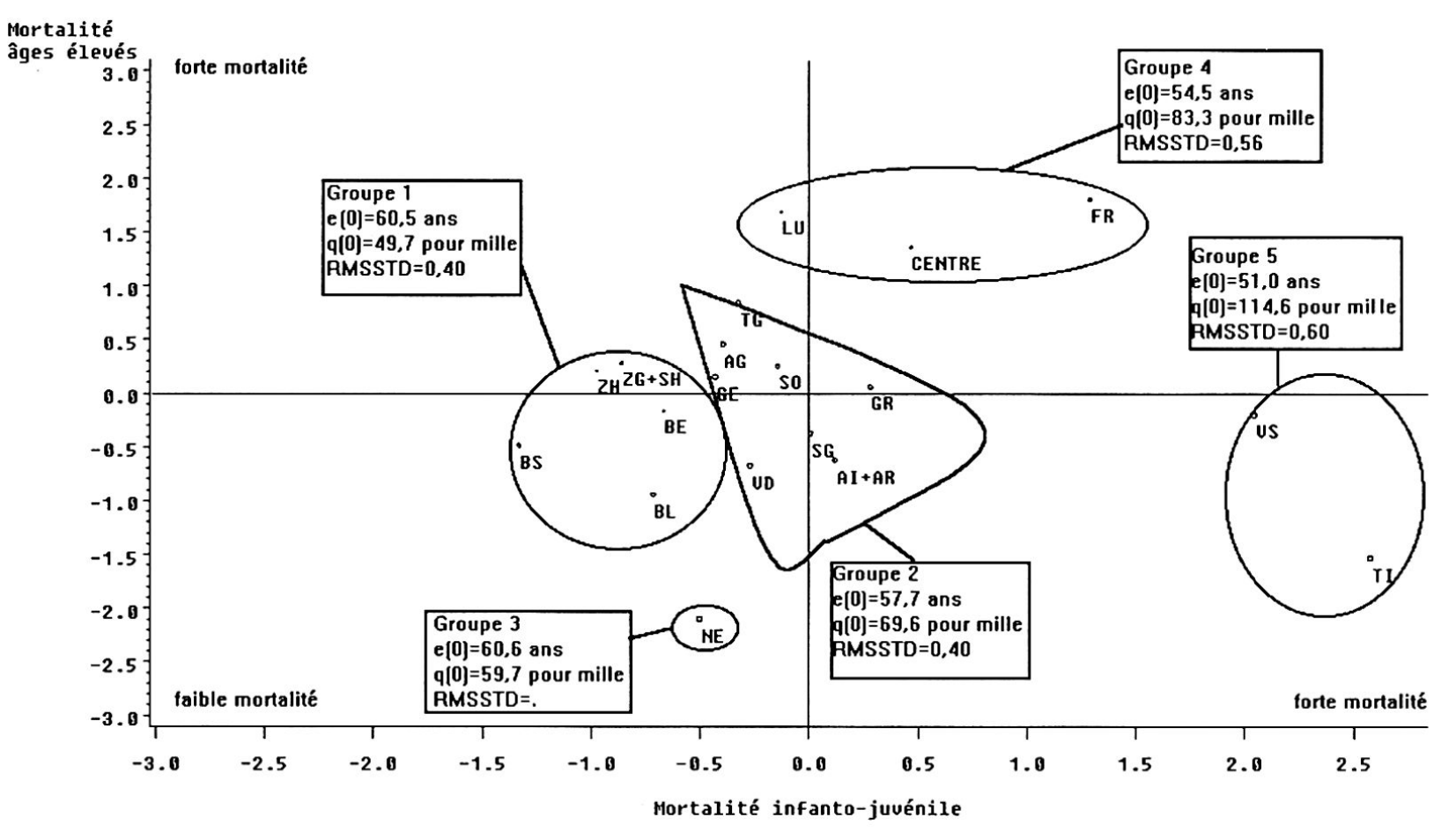

Fig. 4 Confrontation des résultats de l'analyse en composantes principales et de la classification. Position des cantons et groupes de cantons sur le premier plan factoriel, hommes, 1920/21. 


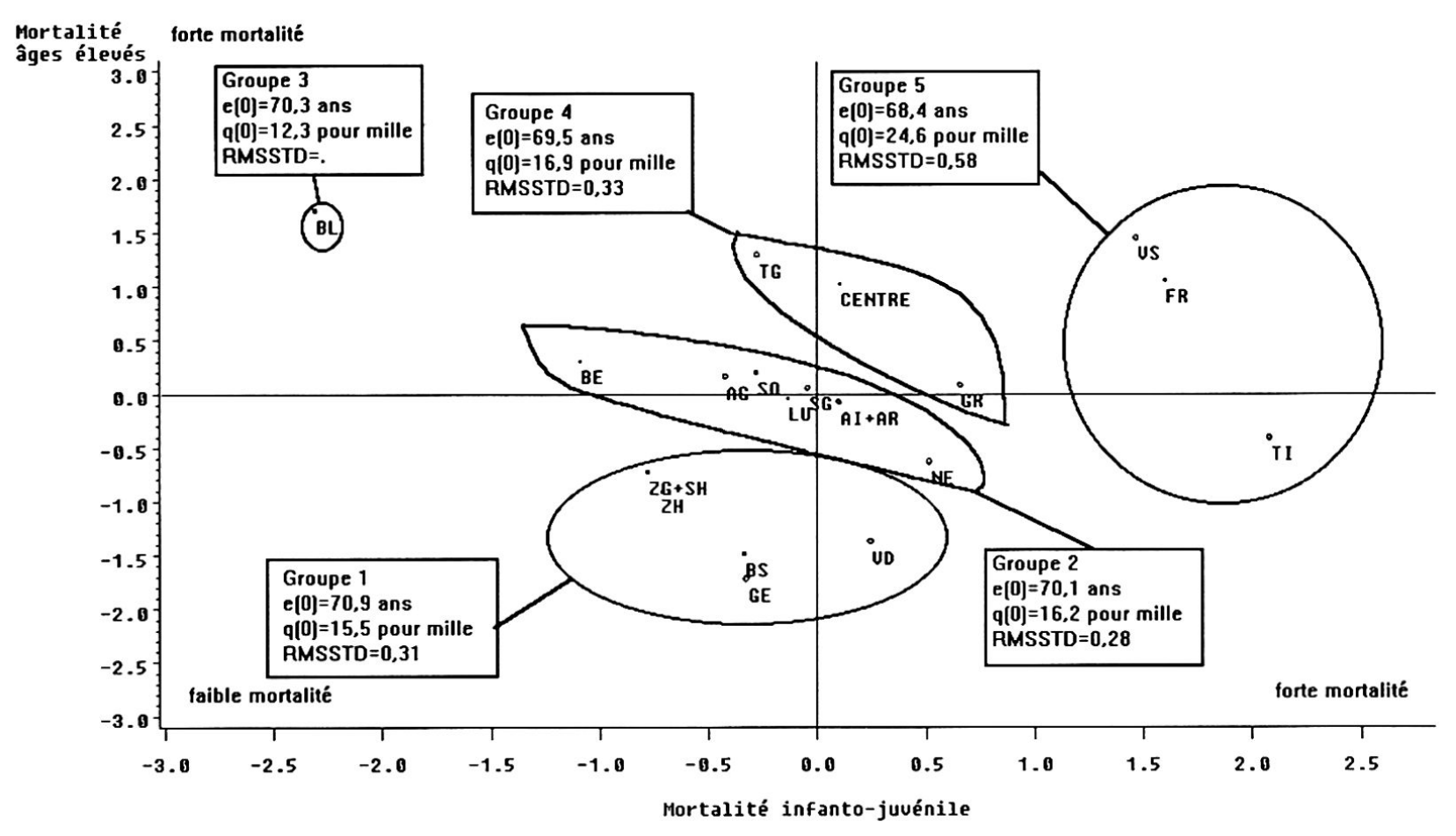

Fig. 5 Confrontation des résultats de l'analyse en composantes principales et de la classification. Position des cantons et groupes de cantons sur le premier plan factoriel, hommes, 1969/72.

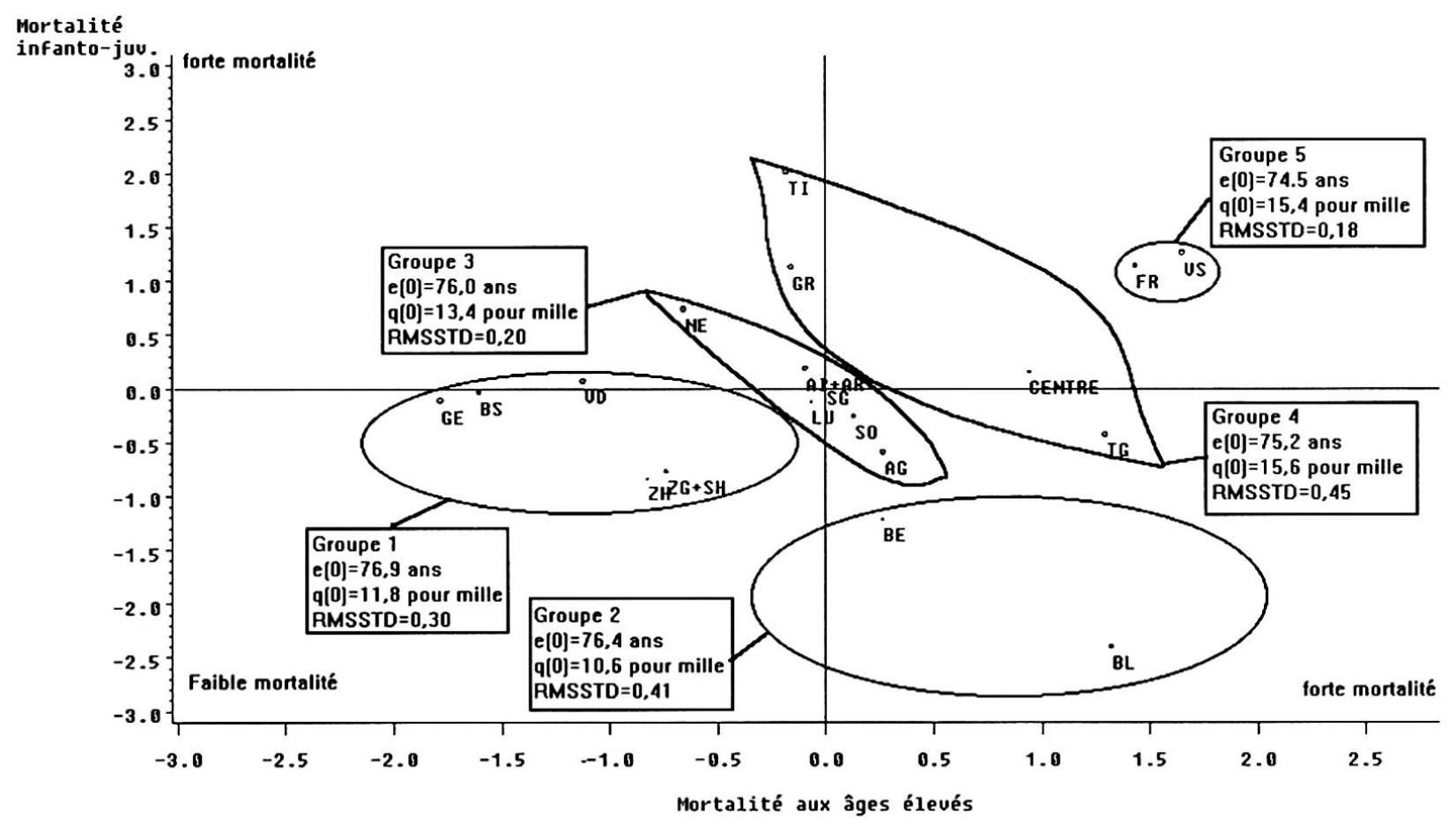

Fig. 6 Confrontation des résultats de l'analyse en composantes principales et de la classification. Position des cantons et groupes de cantons sur le premier plan factoriel, hommes, 1969/72. 


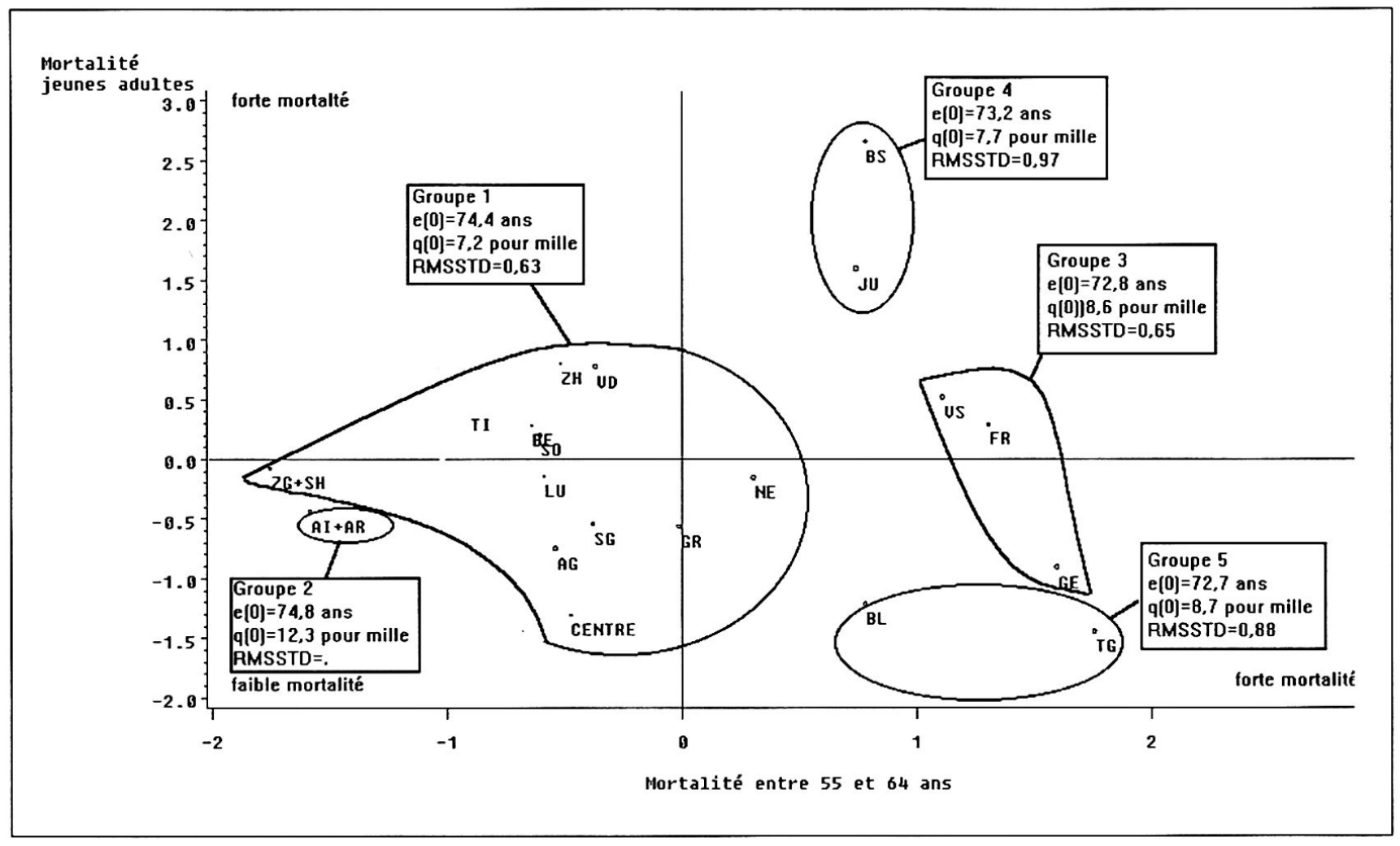

Fig. 7 Confrontation des résultats de l'analyse en composantes principales et de la classification. Position des cantons et groupes de cantons sur le premier plan factoriel, hommes, 1988/93.

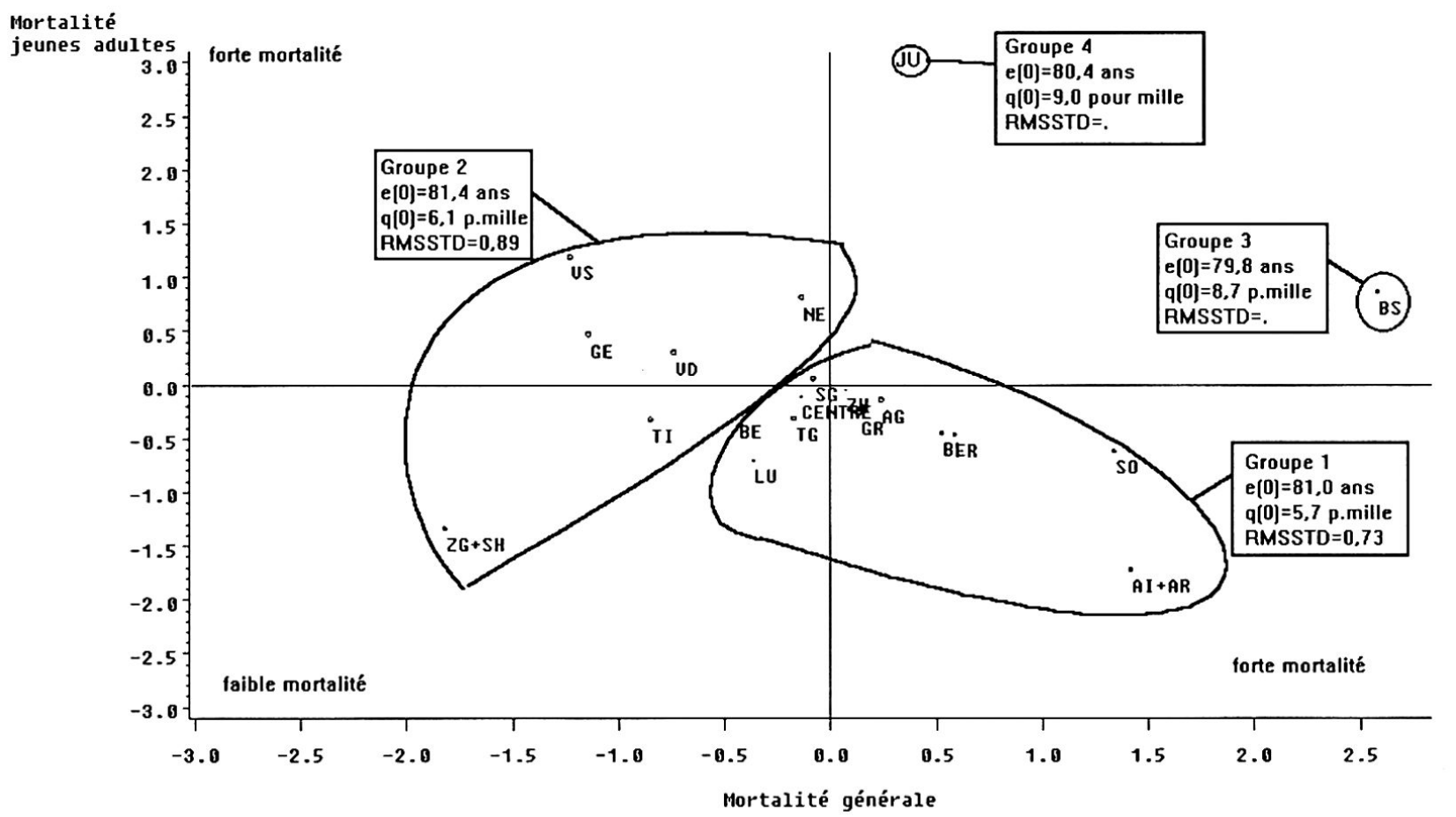

Fig. 8 Confrontation des résultats de l'analyse en composantes principales et de la classification. Position des cantons et groupes de cantons sur le premier plan factoriel, hommes, 1988/93. 


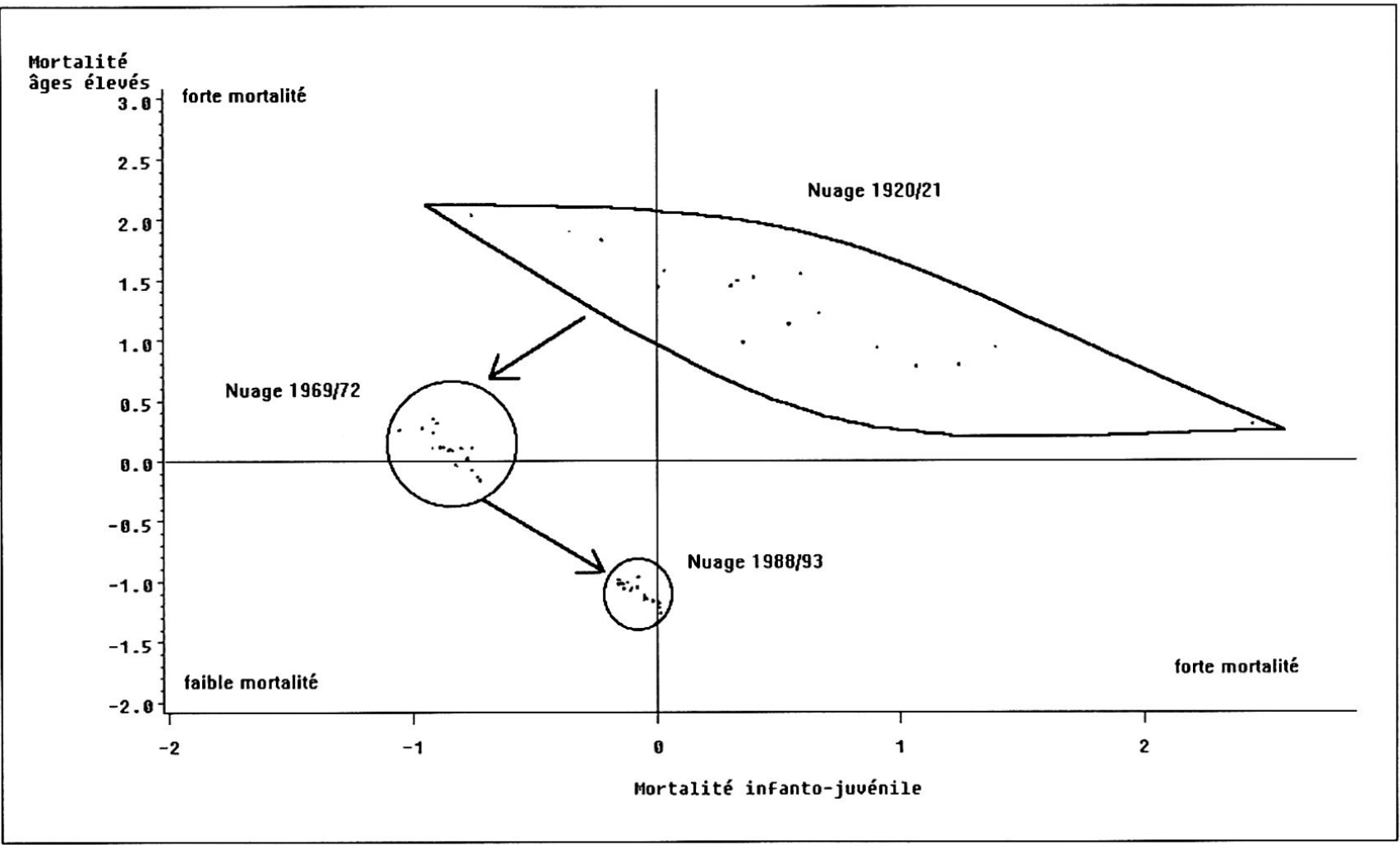

Fig. 9 Position des cantons et groupes de cantons sur le premier plan factoriel, hommes, de 1920/21 à 1988/93.

Mortalité

infanto-juvénile

3.0- Forte mortalité

Nuage 1920/21

Forte mortalite
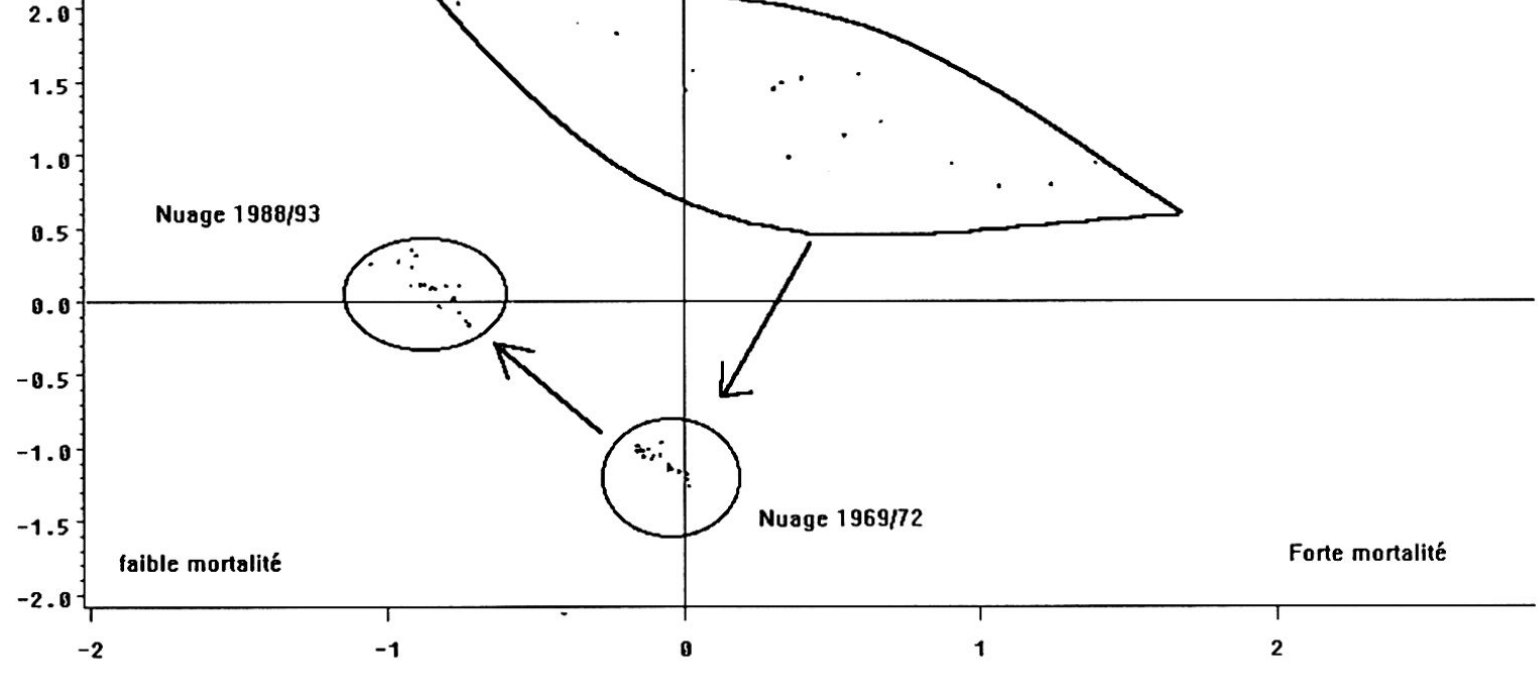

Mortalité aux âges élévés

Fig. 10 Position des cantons et groupes de cantons sur le premier plan factoriel, femmes, de 1920/21 à $1988 / 93$. 
jeunes et aux âges avancés. Chez les femmes, ils forment une classe très homogène. Entre ces deux extrêmes, les cantons sont classés en trois groupes relativement homogènes, selon le niveau de mortalité des personnes d'âge mûr.

Pour 1988/93, un regroupement des cantons en plusieurs classes est difficile. Les schémas de mortalité cantonales sont devenus très homogènes, si bien qu'une détermination statistique de catégories n'a plus grand sens. Ainsi, les valeurs du RMSSTD prises pour les différents groupes définis ( 5 chez les hommes, 4 , dont deux cantons individuels, chez les femmes) sont particulièrement élevées. Le pourcentage de variance conservée par le regroupement des cantons est relativement faible durant cette dernière période, $63,6 \%$ pour les hommes et $38,3 \%$ seulement pour les femmes.

La corrélation entre les variables de mortalité par groupe d'âges est faible, ainsi la capacité explicative du premier plan factoriel est fortement réduite: La part de la variance expliquée par les deux premiers axes est de $60,9 \%$ pour les hommes et de $48,6 \%$ pour les femmes. Un facteur traduit la mortalité des jeunes adultes, l'autre facteur la mortalité entre 55 et 64 ans (chez les hommes) ou la mortalité générale (chez les femmes).

Les résultats sont difficilement interprétables étant donné la faible part de la variance expliquée par les deux premiers axes. On note toutefois que, tant chez les hommes que chez les femmes, certains cantons paraissent conserver des spécificités de mortalité, alors même que les disparités diminuent. Il s'agit du Jura et de BâleVille principalement.

L'ACP prenant en considération les données des trois dates présente les changements de la mortalité durant le siècle (figures 9 et 10 ) et le cheminement des cantons dans un espace géographique représentant à la fois la structure de la mortalité et le temps. Après rotation, le premier facteur explique $49,8 \%$ de la variance totale pour les femmes, et $51,7 \%$ pour les hommes. Il reflète principalement la mortalité des enfants chez les hommes et la mortalité aux âges élevés chez les femmes. Le deuxième facteur explique respectivement $49,7 \%$ (femmes) et $47,3 \%$ (hommes) de la variance totale. Il est saturé, pour les femmes, par les indicateurs de la mortalité infanto-juvénile et, pour les hommes, par les indicateurs de la mortalité aux âges élevés. Sur ces figures, la réduction des disparités de mortalité apparaît clairement, la taille du nuage formé par les observations de chaque groupe de tables a fortement diminué. Tant chez les hommes que chez les femmes, les cantons se sont généralement déplacés du coin supérieur droit vers le coin inférieur gauche entre 1920/21 et 1969/72, traduisant un spectaculaire déclin de la mortalité infantile, ainsi qu'une diminution de la mortalité pour la population âgée. De 1969/72 à 1988/93, l'ACP montre surtout une diminution de la mortalité des personnes âgées, la mortalité aux autres âges augmente légèrement, sous l'effet de l'accroissement de la mortalité des jeunes âges adultes.

\subsection{Approche démographique - une analyse des disparités spatiales}

La décomposition des écarts entre les tables présentant le niveau d'espérance de vie à la naissance le plus faible, respectivement le plus élevé, renseigne sur les âges auxquels attribuent ces disparités (tableau 3). En 1920/21, l'écart de durée de vie entre les cantons ayant les valeurs extrêmes (plus de 10 ans d'écart entre le Tessin et Neuchâtel chez les hommes, entre le Tessin et Bâle-Ville chez les femmes) était dû, pour plus de $70 \%$, à la mortalité infanto-juvénile. En 1969/72, la contribution relative des jeunes âges aux disparités a fortement diminué. En revanche, les âges actifs (35 à 64 ans) et élevés (65 ans et plus) jouent un rôle déterminant sur les disparités cantonales. Pour les âges supérieurs à 64 ans, des disparités, inexistantes au début du siècle, émergent clairement, tant chez les hommes que chez les femmes. Ainsi, dans un régime de mortalité élevée, la mortalité des enfants explique l'essentiel des disparités, tandis que dans un régime de faible mortalité, la population âgée paraît responsable de la plus grande partie de ces disparités.

\section{Discussion}

Le niveau et l'évolution de la mortalité pour la Suisse ont déjà donné lieu à quelques études explicatives historiques (CALOT 1998; PENG FEI et al. 1997; GUBÉRAN 1979, 1980), rendues possibles par la disponibilité de données pour l'ensemble du siècle. En revanche, bien que des tables de mortalité cantonales ou régionales aient été publiées régulièrement depuis le milieu du siècle (BFS 1951; NEURY 1969; OFS 1996b) l'évolution des disparités cantonales de mortalité n'avait jamais été décrite sur une longue période. La présente analyse s'inscrit dans ce contexte et recourt simultanément à une approche statistique et à une approche démographique, afin d'étudier l'évolution de la mortalité et des disparités entre cantons.

La période considérée et sa subdivision en deux étapes distinctes - définies selon des considérations portant sur les conditions sociosanitaires prévalant et leur influence sur la mortalité - permet de comparer l'évolution des disparités cantonales dans deux périodes d'évolution: la première période est caractérisée par une forte diminution de la mortalité, la seconde par une modification du schéma de la mortalité par âge. La première période correspond à une forte diminution des disparités cantonales de mortalité, principalement en raison de la diminution des disparités de mortalité infantile, la seconde coïncide avec une certaine homogénéisation des valeurs cantonales et un probable retournement des disparités cantonales au profit des cantons ruraux et du Tessin, et au détriment des villes et des cantons de montagne. Cette cassure dans les tendances de la mortalité et des disparités régionales est observable tant par l'analyse statistique multidimensionnelle que par l'application d'une méthode typiquement démographique. 
Tableau 3: Différences d'espérance de vie entre les cantons caractérisés par la plus forte et la plus faible mortalité et répartition de ces différences selon la contribution respective de chaque groupe d'âges. $1920 / 21,1969 / 72$ et $1988 / 93^{1}$

\begin{tabular}{|c|c|c|c|c|c|c|c|c|c|c|c|c|}
\hline \multirow[b]{3}{*}{ Cantons, période } & \multirow{2}{*}{\multicolumn{2}{|c|}{$\begin{array}{l}\text { Différence } \\
\text { totale }\end{array}$}} & \multicolumn{10}{|c|}{ Contribution respective des différents groupes d'âges } \\
\hline & & & \multicolumn{2}{|c|}{$0-4$ ans } & \multicolumn{2}{|c|}{$5-19$ ans } & \multicolumn{2}{|c|}{$20-34$ ans } & \multicolumn{2}{|c|}{$35-64$ ans } & \multicolumn{2}{|c|}{65 ans et plus } \\
\hline & années & $\%$ & années & $\%$ & années & $\%$ & années & $\%$ & années & $\%$ & années & $\%$ \\
\hline Hommes, entre: & & & & & & & & & & & & \\
\hline essin & & & & & & & & & & & & \\
\hline $1920 / 21$ & 10,2 & 100,0 & 7,4 & 72,0 & 1,1 & 10,9 & 1,0 & 10,2 & 0,7 & 7,1 & $-0,0$ & $-0,2$ \\
\hline Genève et Valais & & & & & & & & & & & & \\
\hline 1969/72 & 2,9 & 100,0 & 0,6 & 21,2 & 0,2 & 6,1 & 0,3 & 10,2 & 1,1 & 38,9 & 0,7 & 23,5 \\
\hline $\begin{array}{l}\text { Zoug/Schaffhouse } \\
\text { et Fribourg, 1988/93 } \\
\text { Femmes, entre: }\end{array}$ & 2,2 & 100,0 & 0,2 & 10,7 & 0,2 & 8,1 & 0,0 & 2,9 & 1,3 & 57,4 & 0,5 & 20,9 \\
\hline Bâle-Ville et Tessin & & & & & & & & & & & & \\
\hline $\begin{array}{l}\text { 1920/21 } \\
\text { Genève et Valais }\end{array}$ & 11,9 & 100,0 & 8,5 & 71,6 & 1,5 & 12,5 & . & 10,7 & 0,7 & 6,2 & $-0,1$ & $-1,0$ \\
\hline 1969/72 & 2,6 & 100,0 & 0,3 & 12,2 & 0,2 & 6,0 & 0,3 & 10,8 & 1,0 & 37,4 & 0,8 & 33,4 \\
\hline $\begin{array}{l}\text { Tessin et Bâle-Ville } \\
\text { 1988/93 }\end{array}$ & 2,0 & 100,0 & 0,2 & 12,0 & $-0,0$ & $-1,1$ & 0,2 & 10,5 & 0,8 & 41,5 & 0,8 & 37,1 \\
\hline
\end{tabular}

${ }^{1}$ Contributions respectives obtenues selon la méthode d'Ariaga (1984)

Durant la première période (entre 1920/21 et 1969/72), le progrès d'espérance de vie a été spectaculaire. L'espérance de vie à la naissance augmente de près de 16 ans chez les hommes et de près de 19 ans chez les femmes. Cette évolution a débuté bien avant, ${ }^{3}$ mais elle s'est accélérée dès la fin de la Première Guerre mondiale (OFS 1996b). Elle s'explique principalement par la très forte diminution de la mortalité infantile et juvénile, qui a concerné surtout les générations nées entre les deux conflits mondiaux (OFS 1998). Les découvertes médicales n'ont pas l'exclusivité de cette diminution de la mortalité infantile, amorcée bien avant la découverte des antibiotiques en 1948, étape considérée pourtant comme un progrès médical essentiel.

Si la disponibilité et l'usage des antibiotiques ne paraissent pas avoir modifié le rythme d'évolution de la mortalité des jeunes enfants, les médicaments développés pour lutter contre la tuberculose, ainsi que le vaccin contre la poliomyélite et une meilleure hygiène paraissent en revanche avoir eu une influence directe sur ces maladies qui concernent les jeunes adultes (GUBÉRAN 1980).

Cette étape de progrès coïncide également avec une très forte diminution des disparités cantonales. Dans les années 1920, le pays était caractérisé par de profondes différences socio-économiques, entre les régions en extention (villes et régions industrielles) et d'autres régions en perte de vitesse. La déterioration de la conjoncture économique a accentué ce phénomène.

Il est ainsi probable que les disparités de risque de mortalité caractérisant les années 1920, notamment en matière de mortalité infantile, ont trouvé leur source dans les différences socio-économiques caractéristiques d'un pays aux multiples visages. L'uniformisation des niveaux de risque qui marque le milieu du siècle est quant à elle très probablement provoquée par la conjonction de trois facteurs:

a) Les rythmes de développement socio-économiques ont été dans le sens d'une uniformisation. Dans le même élan, le «retard» observé dans la transition démographique des cantons de la Suisse méridionale, notamment le Tessin et le Valais (voir à ce propos BLANC 1990), a été rattrapé, en grande partie par un rythme de diminution beaucoup plus élevé de la mortalité infantile.

b) L'association entre le niveau socio-économique d'une région et la mortalité a été moins forte, en raison notamment des progrès en matière de médecine et d'hygiène. En d'autres termes, alors qu'au début de la période étudiée, la mortalité (notamment la mortalité infantile) était très fortement associée aux conditions économiques et sociales d'une région, cinquante ans plus tard l'association entre les deux phénomènes était bien moins étroite, une autre série de facteurs - sanitaires, comportementaux ou sociaux - réduisant cette association causale.

c) Enfin, dans un régime de faible mortalité, qui caractérise aujourd'hui la Suisse, les disparités de risque de décès ont une influence beaucoup moins spectaculaire sur l'espérance de vie à la naissance que dans un régime de forte mortalité. Pour donner un exemple, une différence de $50 \%$ du niveau de mortalité infantile entre deux cantons aura un impact élevé, en terme de durée moyenne de vie, lorsque ce niveau se situe autour de 80 décès pour 1000 enfants nés vivants (comme c'était 
le cas au début des années 1920). Il aura un impact négligeable lorsque ce niveau se situe à 15 décès pour 1000 (niveau observé vers 1970).

La seconde étape étudiée (1969/72 à 1988/93) est encore mal perçue, à de nombreux points de vue. Elle coïncide, dans de nombreux pays, par une forte diminution du rythme de progrès (dans le nord de l'Europe), voire une réduction de l'espérance de vie à la naissance (dans les pays de l'Europe de l'Est). Pour l'ensemble de la Suisse, l'espérance de vie augmente, à un rythme soutenu, quoique légèrement plus faible qu'au cours des décennies précédentes. La caractéristique essentielle de cette période est la modification du profil par âge de la mortalité, principalement pour les hommes. L'augmentation de la mortalité des jeunes adultes contraste en effet avec la diminution de la mortalité des enfants et des personnes âgées de 35 ans et plus. Cette augmentation de la mortalité des jeunes adultes se rencontre dans tous les cantons pour les hommes et dans les cantons urbains pour les femmes (OFs 1996b). Elle a favorisé l'émergence d'une situation dans laquelle la mortalité urbaine est parfois supérieure à celle rencontrée dans les régions rurales, excepté les cantons de montagne (Jura et Valais principalement).

Les disparités cantonales, qui ont encore légèrement diminué au cours de ces 20 dernières années, s'expliquent essentiellement par les âges supérieurs à 35 ans. Ainsi, d'une situation où la mortalité infantile et juvénile expliquait la part essentielle des disparités de durée de vie, on passe à une situation dans laquelle la mortalité des adultes et personnes âgées provoque l'essentiel de ces disparités. Les cancers, le SIDA, les cirrhoses et, dans les régions de montagne, les accidents expliquent pourquoi dans certains cantons on meurt plus vite que dans d'autres (OFS 1997b). Ces causes de décès sont fortement associées aux comportements de vie, notamment le tabagisme (WANNER 1997). Les inégalités cantonales en matière de caractéristiques socio-économiques ou sanitaires et de revenu restent aujourd'hui encore importantes (OFs 1997c). Elles ne se traduisent plus par des disparités de mortalité, mais plus probablement par des disparités de morbidité, pour lesquelles l'information statistique fait le plus souvent défaut.

Ainsi, d'une situation caractérisée par de fortes disparités cantonales de mortalité probablement explicables par le contexte socio-économique, on est passé progressivement à une situation marquée par de faibles disparités de mortalité, attribuables essentiellement au mode de vie.

En conclusion, il faut noter que cette homogénéisation des valeurs de l'espérance de vie n'est pas spécifique à la Suisse, puisqu'elle s'observe également lorsque l'on compare l'évolution de l'espérance de vie entre les différents pays ou groupes de pays européens (CASELLI et al. 1995). Par ailleurs, il est à prévoir que les différences cantonales de durée moyenne de vie seront déterminées, au cours des prochaines décennies, par l'évolution des comportements de risque et des disparités régionales en la matière.

\section{Notes}

${ }^{1}$ Le choix du nombre d'années pris en compte dans l'élaboration de la table de mortalité dépend essentiellement du nombre de décès nécessaires à ce calcul.

2 Root-mean-square standard deviation, dont la valeur se situe entre 0 et 1 . Plus le RMSSTD est proche de 0 , plus la classe comprend des observations homogènes.

${ }^{3}$ Les données recueillies au niveau du pays montrent que dans les années 1870 déjà, la mortalité était en diminution.

\section{Bibliographie}

ANSON, J. (1993): Regional mortality differences in Britain, 1931-87: a two-dimensional analysis, Journal of Biosocial Science, 25 (3), 383-395.

ARRIAGA, E. E. (1984): Measuring and explaining the change in life expectancies, Demography, 21 (1), p. 83-96. BLANC, O. (1990): Les supports et les résultats démographiques, économiques et sociaux, Dans: J.-B. Racine, C. Raffestin, Nouvelle géographie de la Suisse et des Suisses, Lausanne, Payot.

Bureau fédéral de statistique (1951): Tables de mortalité de la population suisse $1931 / 41$ et 1939/44, Berne.

CALOT, G. (1998): Deux siècles d'évolution démographique en Suisse, Tableaux commentés, Berne, OFS, ODE, 1998.

CASELLI, G. (1991): Health transition and cause-specific mortality, In: R. Schofield, D. Reher, A. Bideau, The decine of mortality in Europe, Clarendon Press, Oxford, England, 68-96 CASELLI, G.; EGIDI, V. (1981): A new insight into morbidity and mortality transition in Italy, Genus, 47 (3-4), 1-29.

CASELLI, G.; MESLÉ, F.; VALLIN, J. (1995): Le triomphe de la médecine. Evolution de la mortalité en Europe depuis le début du siècle, Paris: INED, Dossiers et recherche, no 45. COALE, A. J., et DEMENY, P. (1966): Regional Model Life Tables and Stable Population, Princeton, N. J.: Princeton University Press.

Commission fédérale Vieillir en Suisse (1995): Vieillir en Suisse. Bilan et perspectives. Rapport de la commission fédérale, Office central fédéral des imprimés et du matériel, Berne. GUBÉRAN, E. (1979): Tendances de la mortalité en Suisse 1951-1977. Principales causes de décès, Schweizerische Medizinische Wochenschrift, 109, 1465-1471.

GUBÉRAN, E. (1980): Tendances de la mortalité en Suisse. 2. Maladies infectieuses 1876-1977, Schweizerische Medizinische Wochenschrift, 110, p. 574-583.

HAUG, W. (1995): La révolution grise: les transformations démographiques et leur signification pour la société moderne, Démos 1995/4

HÖPFINGER, F. (1995): La structure par âge et par état civil de la population résidante de la Suisse: Evolution et perspective, Dans: Office fédéral de la statistique, Recensement fédéral de la population 1990. La population en Suisse. Structure et dynamique spatiale, Berne, OFS, 7-59.

KUNST, A. E.; LOOMAN, C. W.; MACKENBACH, J. P. (1991): Are regional mortality patterns in the Netherlands culturally determined?, Espace, Populations, Sociétés (1), 85-91.

NEURY, J.-F. (1969): Mortalités cantonales en Suisse: Estimation de l'espérance de vie à la naissance, Informations statistiques, Genève, $17 \mathrm{p}$.

Office fédéral de la statistique, Association suisse des registres des tumeurs (1984): La distribution géographique de 
la mortalité cancéreuse en Suisse 1979/81, Office fédéral de la statistique, 119 e fascicule, Berne.

Office fédéral de la statistique (1987): Répartition géographique des principales causes de décès en Suisse. Berne.

Office fédéral de la statistique (1996a): Les scénarios de l'évolution démographique de la Suisse 1995-2050, Berne.

Office fédéral de la statistique (1996b): Tables de mortalité pour la Suisse 1988/1993, Berne.

Office fédéral de la statistique (1997a): Atlas structurel de la Suisse, Neue Zürcher Zeitung, Zurich.

Office fédéral de la statistique (1997b): Indicateurs démographiques cantonaux. L'évolution démographique récente dans les cantons, OFS, Berne.

Office fédéral de la statistique (1997c): Annuaire statistique de la Suisse, Neue Zürcher Zeitung, Zurich.

Office fédéral de la statistique (1998): Tables de mortalité longitudinales de la Suisse. Générations 1880-1980, OFS, Berne.

OMRAN, A. R. (1982): Epidemiological transition. 1 Theory, In: ROSS, J. A. (eds.), International encyclopedia of population, vol. 1, New York, Free Press, 1982, 172-175.

Organisation mondiale de la santé (1985): Les buts de la Santé pour tous. Buts de la stratégie régionale européenne de la Santé pour tous, Copenhague, OMS.
PENG FEl; WANNER, PH.; COTTER, S. (1997): Approche dynamique de la différence d'espérance de vie entre hommes et femmes en Suisse, entre 1910/11 et 1988/93. Une application des méthodes de décomposition de Pollard et Arriaga, Revue suisse d'Economie politique et de statistique, 133 (4) $741-754$.

SAS Institute Inc. (1989): SAS/STAT user's guide, version 6, fourth Edition, Cary NC.

SHYROCK, H. S., et SIEGEL, J. S. (1971): The Methods and Materials of Demography, Washington, Vol. 2.

VALKOVICS, E. (1996): Quelques considérations sur la nature des transformations des fonctions de la table de mortalité sous l'influence du changement des taux de mortalité par âge et par causes, Dans: AIDELF, Morbidité, mortalité: problèmes de mesure, facteurs d'évolution, essai de prospective, Sinaia, 3-6 septembre 1996.

VOLLÉ, M. (1985): Analyse des données, Paris, Economica. WANNER, PH.; PENG FEl; COTTER, S.: Mortalité par âge et cause de décès en Suisse: une analyse des disparités cantonales durant la période $1978 / 83$ et $1988 / 93$, Revue européenne de démographie, 1997 (4).

WANNER, PH. (1997): L'impact du tabagisme sur la mortalité des Suisses et sur leur durée de vie en 1988/1993, Médecine sociale et préventive, 42, 223-229. 